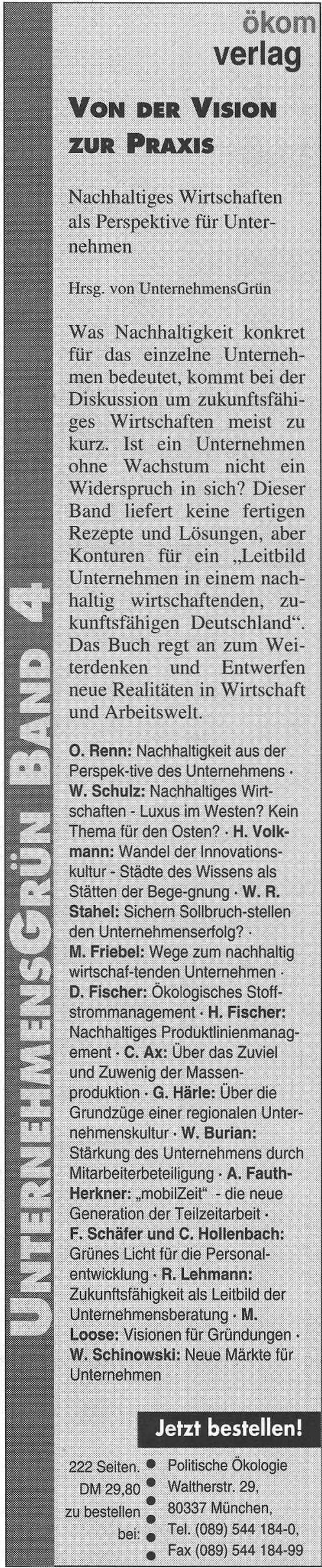

Das neve Gutachten des Wissenschaftlichen Beirats Globale Umweltweränderungen (WBGU)

\title{
Wege aus der Wasserkrise
}

\author{
Wenn von globalen Umweltproblemen die Rede ist, steht meist der Treibhaus- \\ effekt im Brennpunkt der Aufmerksamkeit. Der WBGU macht am Beispiel Süß- \\ wasser deutlich, daß auch andere schleichende Umweltveränderungen in \\ Zukunft für große Konflikte sorgen können.
}

$\mathrm{H}$ Von Jan Nill eute leben rund 2 Milliarden Menschen ohne Zugang zu sauberem Trink- und Sanitärwasser, weltweit werden nur 5 Prozent der Abwässer gereinigt. Infolgedessen leidet jeder zweite Mensch in den Entwicklungsländern an einer wasserbedingten Krankheit. Auch ist Süßwasser der wichtigste limitierende Faktor für die Nahrungsmittelproduktion. Prognostiziert wird eine weltweite Steigerung der Wassernachfrage, wobei sich in den nächsten 30 Jahren allein die Wasserentnahme durch die Industrie verdreifachen soll. Dies birgt den Keim einer globalen sozialen und ökologischen Krise in sich. Internationale Konflikte um die kostbare Ressource sind in vielen Teilen der Welt vorauszusehen, insbesondere im Nahen Osten.

Der WBGU analysiert in seinem jüngst veröffentlichten Jahresgutachten 1997 (1) die Ursachen der Krise anhand des Syndrom-Ansatzes (vgl. hierzu auch den Beitrag von Mogalle/ Minsch in diesem Heft). Drei der 16 „Erdkrankheiten“ werden als besonders relevant angesehen: Das Grüne-Revolution-Syndrom, d.h. die auf Technisierung basierende Modernisierung der Landwirtschaft, das Favela-Syndrom, also die mit Armut und Umweltzerstörung verbundene ungeregelte Urbanisierung, und das Aralsee-Syndrom, das die Problematik zentral geplanter, großtechnischer Wasserbauprojekte beschreibt. Ein allen Syndromen gemeinsames ,Schlüsselthema" ist die Schädigung von Süßwasserlebensräumen und der angrenzenden Biotope durch menschliche Einflüsse über ihre Belastungsgrenzen hinaus.

Als allgemeinen Lösungsansatz zur Vermeidung einer Krise schlägt der Beirat ein LeitplankenModell vor: Ein soziokultureller und ökologischer Rahmen dient als Grenze für die nach Effizienzkriterien erfolgende wirtschaftliche Nutzung des Wassers. Die ökologischen Leitplanken orientieren sich an den Erneuerungs- und Selbstreinigungsraten des Wassers und am Schutz wertvoller Biotope. Ausgangspunkt der sozialen Leitplanken ist die Sicherung der individuellen Grundversorgung, wobei länder- und kulturspezifische Süßwasserbedarfe und internationale Gerechtigkeitsgrundsätze zu berücksichtigen sind.

Zur Umsetzung der so abgeleiteten Leitlinien entwirft der WBGU eine globale Strategie mit den Elementen internationaler Konsens, Weltwassercharta und internationaler Aktionsplan. Es wird eine Palette von fallspezifisch anzuwendenden Instrumenten vorgeschlagen. Besonders von Wasserkrisen betroffene Staaten sollen besser unterstïtzt werden. Als Finanzierungsinstrumente werden u.a. eine Verknüpfung von wasserpolitischen Programmen mit einer Schuldenreduzierung und ein von einem ,Welt-Wasserpfennig“ gespeister globaler Wasserfonds genannt.

Dem WBGU gebiihrt das Verdienst, auf ein vernachlässigtes Umweltproblem nachdrücklich aufmerksam gemacht zu haben. Das Gutachten läßt sich zugleich als Aufforderung an die Forschung verstehen, Konzepte für die Präzisierung von Leitplanken zu entwickeln. Thre praktische Verankerung ist angesichts der bestehenden Nutzungskonflikte ein sehr ehrgeiziges Ziel. Die historische Erfahrung in den Industrieländern gibt nur begrenzt zu Hoffnung Anlaß, wie das Beispiel der Zerstörung von Ökosystemen an großen Flüssen u.a. durch Staudämme zeigt.

\section{Anmerkung}

(1) Wissenschaftlicher Beirat der Bundesregierung Globale Umweltverönderungen: Welt im Wandel. Wege zu einem nachhaltigen Umgang mit Süßwasser. Springer-Verlag, Berlin 1998. Der WBGU wurde im Frühjiahr 1992 als unabhöngiges Beratergremium von der Bundesregierung eingerichtet. Bisher erschienen in der Reihe Welt im Wandel folgende Jahresgutachten: Grundstruktur globaler Mensch-Umwelt-Beziehungen (1993), Die Gefährdung der Böden (1994), Wege zur Lösung globaler Umweltprobleme (1995) und Heroustorderung für die deutsche Wissenschaft (1996). Internet: hitp://www.awi-bremerhaven.de./wbgu 
(c) 20I0 Authors; licensee IÖW and oekom verlag. This is an article distributed under the terms of the Creative Commons Attribution Non-Commercial No Derivates License (http://creativecommons.org/licenses/by-nc-nd/3.o/), which permits unrestricted use, distribution, and reproduction in any medium, provided the original work is properly cited. 